\title{
Post-ablation prolongation of atrioventricular nodal refractory period is correlated with long-term success of cryoablation for atrioventricular nodal reentrant tachycardia in the case of the persistence of a residual jump
}

\author{
Joelci Tonet • Antonio De Sisti • \\ Natalia Pardo Restrepo • Denis Raguin • Walid Amara • \\ Manlio F. Márquez • Philip Aouate • \\ Xavier Waintraub • Faouzi Touil • \\ Francoise Hidden-Lucet
}

Received: 12 February 2012 / Accepted: 28 February 2012 / Published online: 5 May 2012

(C) The Author(s) 2012. This article is published with open access at Springerlink.com

\author{
Abstract \\ Purpose A residual slow pathway after successful cryoabla- \\ tion for atrioventricular nodal reentrant tachycardia \\ (AVNRT) is correlated with a higher recurrence rate. We \\ J. Tonet $\cdot$ A. De Sisti $\cdot$ P. Aouate $\cdot$ X. Waintraub $\cdot$ F. Touil $\cdot$ \\ F. Hidden-Lucet \\ Cardiology Institute, Rhythmology Unit, \\ Pitié-Salpêtrière Hospital, \\ 47-83, boulevard de l'Hôpital, \\ 75651 Paris, France \\ N. Pardo Restrepo \\ Universidad CES, \\ Calle 10 A No. 22, \\ Medellín, Colombia

\section{Raguin} \\ Rhythmology Unit, Clinique de l'Europe, \\ Amiens, France \\ W. Amara \\ Cardiology Department, le Raincy-Montfermeil Hospital, \\ Montfermeil, France

\section{F. Márquez} \\ Departamento de Electrofisiología, \\ Instituto Nacional de Cardiología Ignacio Chávez, \\ Juan Badiano 1, Col. Sección XVI, \\ 14080 Mexico City, Mexico \\ J. Tonet $(\bowtie)$ \\ Institut de Cardiologie, Unité de Rythmologie, \\ Hôpital de La Pitié-Salpêtrière, \\ 47-83, Boulevard de l'Hôpital, \\ 75651 Paris, France \\ e-mail: joelci.tonet@psl.aphp.fr
}

described determinants of recurrence in subjects with a residual jump.

Methods We analyzed the data of subjects with acute successful slow pathway cryoablation for AVNRT using a 6mm-tip cryocatheter. Success was defined as AVNRT noninducibility. Patients with no baseline elicitable jump, no inducible AVNRT, and transient first atrioventricular (AV) block at the last site were excluded.

Results From 371 patients who underwent cryoablation from May 2002 to March 2011, 303 fulfilled the entry criteria (mean age, 41 $\pm 16 ; 222$ women). Baseline AV nodal effective refractory period (ERP) was $272 \pm 57 \mathrm{~ms}$, postprocedural 331 $\pm 64(P<0.001)$, and the mean of the difference ( $\triangle$ ERP) $60 \pm$ 41. At the end of the procedure, 64 patients $(21 \%)$ had a residual jump, of whom 22 with a single echo. At 12 months follow-up, the actuarial recurrence-free rate was $70.3 \%$ in patients with a residual jump and $86 \%$ in those without $(P=$ $0.01)$. In patients with a jump, only $\triangle \mathrm{AV}$ nodal ERP was correlated with recurrence $(37 \pm 41$ vs. $68 \pm 47 \mathrm{~ms} ; P<0.04)$ while a single echo was not. The actuarial rate of recurrence was $60.8 \%$ in patients with a $\Delta$ AV nodal ERP $\leq 30 \mathrm{~ms}$ and $18.8 \%$ in those with a $\Delta$ AV nodal ERP $>30 \mathrm{~ms}(P<0.01)$.

Conclusions Suppression of slow pathway conduction is the optimal endpoint for AVNRT cryoablation. A residual jump can be tolerated if AV nodal ERP postcryoablation is prolonged $>30 \mathrm{~ms}$.

Keywords Atrioventricular nodal reentry tachycardia - Cryoablation - Radiofrequency catheter ablation - Slow pathway - Atrial echo · Atrioventricular nodal effective refractory period 


\section{Introduction}

Slow pathway ablation is the treatment of choice for atrioventricular nodal reentrant tachycardia (AVNRT). Cryoablation is effective and safe, but its widespread use seems to be limited by a slightly lower long-term clinical efficacy when compared to radiofrequency (RF) ablation [1]. After an RF procedure, residual conduction through the slow pathway associated with AVNRT non-inducibility does not seem to modify long-term results [2]. Contrarily, the persistence of a residual slow pathway after cryoablation has been correlated with a higher recurrence rate [3-6]. In the present study, we described determinants of recurrence in subjects with a residual jump after successful cryoablation for AVNRT. We focused on correlation of the increment of atrioventricular (AV) nodal effective refractory period (ERP) measured at the end of the procedure with the clinical outcome of these patients.

\section{Methods}

Patients from three French centers (Pitié-Salpêtrière Hospital, Paris; Le Raincy-Montfermeil Hospital, Montfermeil; and Clinic of Europe, Amiens) with successful cryoablation procedures for AVNRT performed with a 6-mm-tip cryocatheter (Freezor Xtra CryoCath ${ }^{\circledR}$ ) were included. All centers adhered to inclusion/exclusion criteria, procedural methods, and endpoints. All subjects gave informed consent for the study. Exclusion criteria were: lack of baseline elicitable jump, non-inducibility of AVNRT at baseline, previous ablation attempts, other associated arrhythmias, underlying heart disease, transient first-degree AV block at the last ablation site, and the need for beta blocking agents.

Procedure A standard electrophysiologic (EP) study was performed in the fasting state without sedation. Antiarrhythmic drugs were discontinued for at least five half-life periods. Dual AV nodal physiology was defined as a $\geq 50 \mathrm{~ms}$ increase in $\mathrm{A} 2 \mathrm{H} 2$ in response to a $10 \mathrm{~ms}$ decrease during A1A2 stimulation [7]. If sustained tachycardia could not be induced, isoproterenol was administered. Slow pathway potentials in the Koch triangle were identified as the target site $[7,8]$ in a zone located anterior to the coronary sinus (CS), slightly below an ideal line delimiting the superior border of the CS ostium; an $\mathrm{A} / \mathrm{V}$ ratio of $\sim 1$ was generally preferred as a target (Fig. 1). Cryomapping was carried out first at a cryocatheter tip temperature of $-30 / 40{ }^{\circ} \mathrm{C}$ for 30 $45 \mathrm{~s}$ to test the cryo-effects on the target sites by using programmed stimulation. Cryoablation $\left(-75 / 80{ }^{\circ} \mathrm{C}\right.$ for $4 /$ $5 \mathrm{~min}$ ) was initiated immediately following successful cryomapping, defined as abolition of the slow pathway or AVNRT noninducibility. If AVNRT remained inducible or

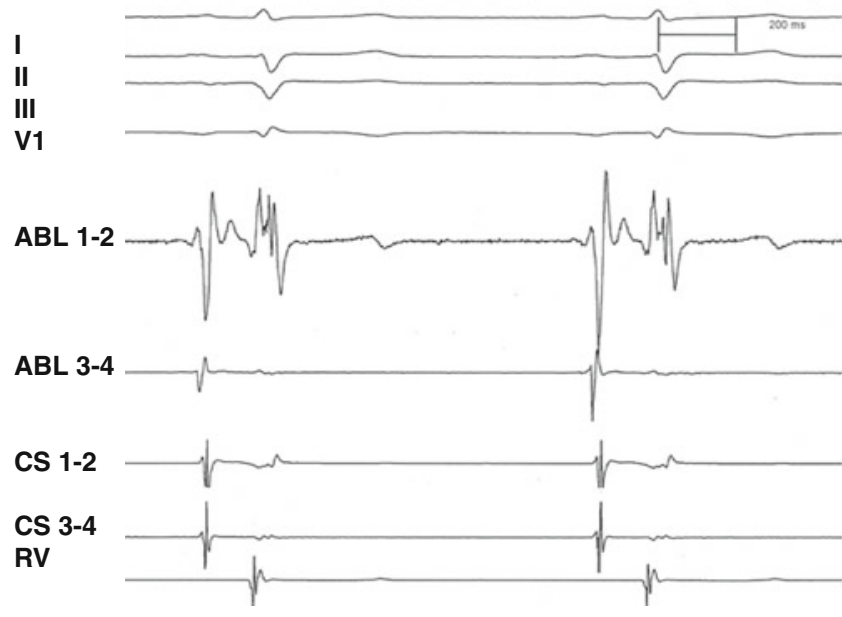

Fig. 1 An example of optimal target site for AVNRT slow pathway cryoablation. Note an $\mathrm{A} / \mathrm{V}$ ratio of about 1 and a stable nodal $\mathrm{AV}$ potential. $A V N R T$ atrioventricular nodal reentry tachycardia, $A / V$ atrioventricular

AV block occurred, cryoablation was stopped and a further sequence of cryomapping and ablation at new target sites was repeated. A freezing-thaw-freezing cycle of cryoablation was not systemically carried out [1]. An EP study was continuously repeated during the waiting period of 3045 min after the last cryoablation to check the procedure's effectiveness, with and without isoproterenol infusion. Success was defined as non-inducibility of AVNRT. A residual slow pathway conduction, associated or not with a reproducible single echo, was permitted at the discretion of the senior operating electrophysiologist. Nodal refractoriness of the fast pathway was not systematically measured in this study. The final postprocedural AV nodal ERP was evaluated before isoproterenol administration. The cryoablation procedures were performed by 10 operators.

Post-ablation management and follow-up After cryoablation, no antiarrhythmic drugs were prescribed. During the follow-up, patients underwent trans-telephonic assessment of symptoms, rest ECG and 24-h Holter recording at 3 months and then every 6 months in our centers or by their referring physicians. Relapse was defined as the recurrence of index arrhythmia-typical symptoms of tachycardia with sudden onset and termination, tachycardia documented by ECG or 24-h Holter recording. The minimum observational period was set at 6 months.

\section{Statistical analysis}

Continuous variables were expressed as mean \pm SD. In the comparison between groups, ANOVA was used for continuous variables, and $X^{2}$ test for discrete variables. Correlation 
between long-term results in the follow-up and the different variables was performed using Cox's regression model for univariate and multivariate analysis. Continuous variables were converted into dichotomic when appropriate. Following univariate analysis, factors with an associated $P$ value $<$ 0.10 were tested in multivariate analysis. A stepwise regression procedure was used to determine independent predictors. The $P$ value for entry or removal of a variable from the regression model was 0.05 and 0.10 , respectively. Actuarial graphs were constructed using the Kaplan-Meier method and differences were evaluated by log-rank test. A $P$ value $<$ 0.05 was considered significant. Statistical analysis was performed using SPSS 19 for Windows.

\section{Results}

\subsection{Study group characteristics}

Among 371 patients who had undergone AVNRT cryoablation from May 2002 to March 2011, 303 of them (mean age, $41 \pm 16$ years; 222 women) fulfilled the entry criteria (Table 1 ). Patients excluded were: 12 for initial unsuccessful procedures, 46 for absence of baseline jump or AVNRT inducibility, and 10 for transient first-degree AV block at the last effective

Table 1 Clinical and procedural characteristics of the study population

\begin{tabular}{ll}
\hline Patients $(n)$ & $303(\%)$ \\
Type of AVNRT & \\
Slow-fast & $284(94)$ \\
Fast-slow & $6(2)$ \\
Slow-slow & $13(4)$ \\
AVNRT cycle length (ms) & $331 \pm 65$ \\
Cryoapplications ( $n$ ) & $6.3 \pm 5.8$ \\
Total cryoapplication time (s) & $1,052 \pm 797$ \\
A/V at last effective site (ratio) & $1 \pm 0.65$ \\
Procedure time (min) & $128 \pm 47$ \\
Fluoroscopy time (min) & $17 \pm 13$ \\
Transient second/third degree AV block at & 16 \\
last site (patients) & \\
AV node ERP (ms) & \\
Baseline ERP & $272 \pm 57$ \\
Post-procedural ERP & $331 \pm 64$ \\
$\Delta$ ERP & $60 \pm 41$ \\
Post-procedural residual jump (patients) & $64(21)$ \\
Residual jump without single echo & 42 \\
Residual jump with echo & 22 \\
Recurrence (patients) & $53(17.5)$ \\
Redo procedure (patients) & $24(7.9)$ \\
\end{tabular}

$A A$ antiarrhythmic, $A V$ atrioventricular, $E R P$ effective refractory period, $\triangle E R P$ difference between baseline and postprocedural ERP ablation site. The contribution of each participating center to the study was: 301 patients from Pitié-Salpêtrière Hospital, 31 from Le Raincy-Montfermeil Hospital, and 30 from the Clinic of Europe.

Previous ineffective drugs in the 303 patients analyzed were $1.2 \pm 1$. Patients had the following AVNRT subtypes: slow-fast AVNRT in 284 patients, fast-slow in six, and slowslow in the remaining 13. AVNRT cycle length was $331 \pm$ $65 \mathrm{~ms}$. The number of cryoapplications was $6.3 \pm 5.8$ per patient. Total cryoapplication time was $1,052 \pm 797 \mathrm{~s}$. The procedure and fluoroscopy times were $128 \pm 47$ and $17 \pm$ 13 min, respectively. The $\mathrm{A} / \mathrm{V}$ ratio at the last effective site was $1 \pm 0.65$. Transient second and third degree AV block at the last ablation site occurred in 16 patients (5.3\%). Baseline AV nodal ERP was $272 \pm 57 \mathrm{~ms}$, postprocedural ERP $331 \pm 64 \mathrm{~ms}(P<0.001)$, and the mean of the difference between baseline and postprocedural ERP ( $\triangle$ ERP) $60 \pm$ $41 \mathrm{~ms}$. A postprocedural residual jump was elicited in 64 patients (21\%), of whom 22 with an associated single echo. During a mean follow-up of $45 \pm 15$ months, 53 (17.5\%) patients had recurrences. Redo procedures were performed in 24 patients $(7.9 \%)$.

\subsection{Predictors of recurrence in the study population}

Recurrence was associated with a residual postprocedural jump ( $18 / 53$ vs. $44 / 250$ patients; $P<0.02)$, and a prolonged total cryoapplication time $(1,389 \pm 884$ vs. $982 \pm 762 \mathrm{~s} ; P<$ 0.001; Table 2, Fig. 2). There was no significant difference concerning other clinical and procedural variables (including $\Delta$ AV nodal ERP) between patients with or without recurrence in the follow-up. At univariate and multivariate analysis, residual jump (univariate analysis HR, 2.05 [95\% CI 0.26-0.77], $P<0.02$; multivariate analysis HR, 0.5 [95\% CI 1.03-3.8], $P<0.04$ ), and total cryoapplication time $>2,000 \mathrm{~s}$ (univariate analysis HR 2.42 [95\% CI 1.29 4.53], $P<0.01$; multivariate analysis HR, 2.16 [95\% CI 1.14-4.08] $P=0.01$ ) were significantly correlated with recurrence. At 12-months follow-up, the actuarial rate of recurrence-free patients was $70.3 \%$ in the group with a residual jump ( 245 patients), and $86 \%$ in those without a residual jump $(P=0.01)$.

4.3 Predictors of recurrence in subjects without a residual slow pathway at the end of the procedure

In the subgroup of patients without a residual jump at the end of the procedure, total cryoapplication time was more prolonged in patients with recurrence $(1401 \pm 855$ vs. $962 \pm$ 753 s; $P<0.005$; HR: 3.14, 95 \% CI: 1.49-6.61; $P<0.005$; Table 2). No significant difference was found in terms of $\Delta$ AV nodal ERP between patients with and without 
Table 2 Procedural characteristics of patients with and without recurrence

\begin{tabular}{|c|c|c|c|c|c|c|c|c|c|}
\hline & \multicolumn{3}{|c|}{ Total study population } & \multicolumn{3}{|c|}{ Subgroup without residual jump } & \multicolumn{3}{|c|}{ Subgroup with residual jump } \\
\hline & $\begin{array}{l}\text { Recurrence } \\
(n=53)\end{array}$ & $\begin{array}{l}\text { No recurrence } \\
(n=250)\end{array}$ & $P$ & $\begin{array}{l}\text { Recurrence } \\
(n=35)\end{array}$ & $\begin{array}{l}\text { No recurrence } \\
(n=204)\end{array}$ & $P$ & $\begin{array}{l}\text { Recurrence } \\
(n=18)\end{array}$ & $\begin{array}{l}\text { No recurrence } \\
(n=46)\end{array}$ & $P$ \\
\hline Cryoapplication time (s) & $1,389 \pm 884$ & $982 \pm 762$ & $<0.001$ & $1,401 \pm 855$ & $962 \pm 753$ & $<0.005$ & $1,384 \pm 969$ & $1,067 \pm 881$ & NS \\
\hline Residual jump & $18(34 \%)$ & $46(18.4 \%)$ & $<0.02$ & - & - & - & - & - & - \\
\hline Associated single echo $(n)$ & $8(15 \%)$ & $14(5.6 \%)$ & $<0.02$ & - & - & - & $8(44.4 \%)$ & $14(30.4 \%)$ & NS \\
\hline$\Delta$ AV ERP (ms) & $40 \pm 5.8$ & $42 \pm 3$ & NS & $66 \pm 35$ & $61 \pm 40$ & NS & $37 \pm 41$ & $68 \pm 47$ & $<0.04$ \\
\hline
\end{tabular}

$A V$ atrioventricular, $E R P$ effective refractory period, $\triangle A V$ node ERP difference between baseline and postprocedural AV nodal ERP

recurrence ( $66 \pm 35$ vs. $61 \pm 40 ; P=\mathrm{NS})$, whatever the cutoff value used (Fig. 3).

4.4 Predictors of recurrence in patients with a residual slow pathway at the end of the procedure

In patients with a residual jump, only $\Delta$ AV nodal ERP ms was significantly shorter in patients with recurrence $(37 \pm 41$ vs. $68 \pm 47 \mathrm{~ms} ; P<0.04$; Table 3 , Fig. 4). There was no significant difference in patients with and without recurrence in terms of total cryoapplication time (1,384 \pm 969 vs. $1,067 \pm 881 \mathrm{~s} ; P=\mathrm{NS})$ and the presence of a single echo ( $8 / 18$ vs. $14 / 46$ patients; $P=\mathrm{NS})$. At 12 -months follow-up, the actuarial rate of recurrence-free patients was $62.9 \%$ in the group with an associated single echo and $74.1 \%$ in those without $(P=\mathrm{NS})$.

Using Cox's model, $\triangle$ AV nodal ERP $\leq 30 \mathrm{~ms}$ was the best predictor of recurrence (HR: 0.25 , CI: $0.09-0.69$; $P<$ $0.01)$. At 12-months follow-up, the actuarial rate of recurrence was $60.8 \%$ in the group with $\mathrm{AV}$ nodal ERP $\leq 30 \mathrm{~ms}$ and $18.8 \%$ in those with AV nodal ERP $>30 \mathrm{~ms}(P<0.01)$.

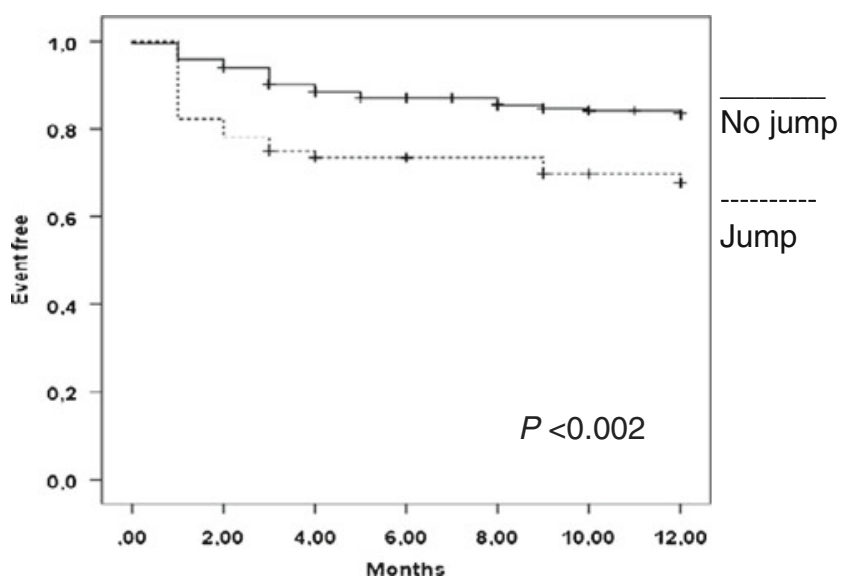

Fig. 2 AVNRT recurrence-free event depending on postprocedural residual slow pathway. $\Delta$ AV nodal ERP: difference between baseline and post-procedural nodal effective refractory period

\section{Discussion}

This study confirms that slow pathway persistence after cryoablation for AVNRT is correlated with a worse longterm outcome [1]. Also, as previously described [6], the persistence of a single echo in patients with a residual jump did not represent an adjunctive risk. A new finding was that the modification of slow pathway properties, specifically the prolongation of AV nodal ERP post-cryoablation, was associated with a better outcome in patients with residual slow pathway conduction. Contrarily, in those with complete slow pathway suppression at the end of the procedure, AV nodal ERP prolongation was not correlated with outcome.

\subsection{Global results for AVNRT cryoablation}

Cryoablation for AVNRT has been the object of numerous studies in the last decade. In a recent review of the literature, we showed [1] in data pooled from 20 published studies including 2,351 patients [3-5, 9-25] that the initial procedural success rate for AVNRT cryoablation was $95 \%$ (range, 85-99 \%), not so far from the acute success described in RF series [26, 27]. Overall, the mean recurrence

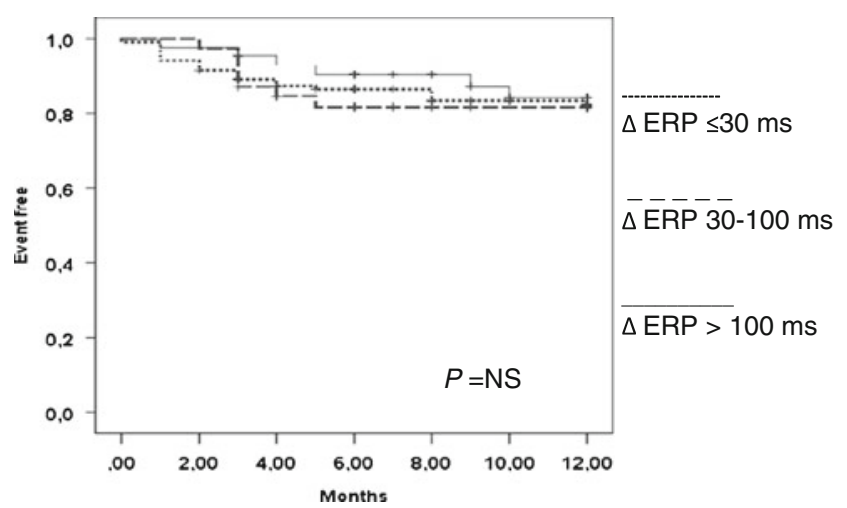

Fig. 3 Patients without postprocedural residual slow pathway. AVNRT recurrence-free event depending on $\triangle$ AV nodal ERP. $A V N R T$ atrioventricular nodal reentry tachycardia, $\triangle A V$ nodal ERP difference between baseline and postprocedural nodal effective refractory period 
Table 3 Predictive factors of recurrence in the study population (303 patients)

\begin{tabular}{|c|c|c|c|c|c|c|}
\hline \multirow[b]{2}{*}{ Variables } & \multicolumn{3}{|c|}{ Univariate analysis } & \multicolumn{3}{|c|}{ Multivariate analysis } \\
\hline & HR & $95 \%$ CI & $\mathrm{p}$ & $\mathrm{HR}$ & $95 \%$ CI & $P$ \\
\hline Cryoapplication time $>2,000 \mathrm{~s}$ (patients) & 2.42 & $1.29-4.53$ & $<0.01$ & 2.16 & $1.14-4.08$ & $=0.01$ \\
\hline Residual jump (patients) & 2.05 & $0.26-0.77$ & $<0.02$ & 0.5 & $1.03-3.28$ & $<0.04$ \\
\hline
\end{tabular}

rate was $11 \%$ (range, $7-19.7 \%$ ), higher than for RF catheter ablation, in which the recurrence rate has been reported as being between 3 and $5 \%[26,27]$.

\subsection{Residual slow pathway in patients treated} by radiofrequency ablation

With RF energy, the persistence of a slow pathway with no more than a single echo can be an acceptable end-point. Recently, Stern et al. [2], in a meta-analysis including 1,204 patients from 10 studies, found that if isoproterenol was systematically used at the end of an RF procedure, no significant difference in recurrence rates could be found between patients with complete slow pathway suppression and those with a residual jump associated or not with a single echo. However, in studies in which isoproterenol was not systematically used, slow pathway suppression was associated with a lower risk of recurrence compared to slow pathway modifications.

\subsection{Residual slow pathway in patients treated} by cryoablation

Globally, in studies including patients treated with cryoenergy, isoproterenol was frequently used at the end of the procedure, systematically or limited to patients with a residual slow pathway [1]. In the series of Gupta et al. [3], a single echo was induced in 20/71 (28\%) patients in the RF

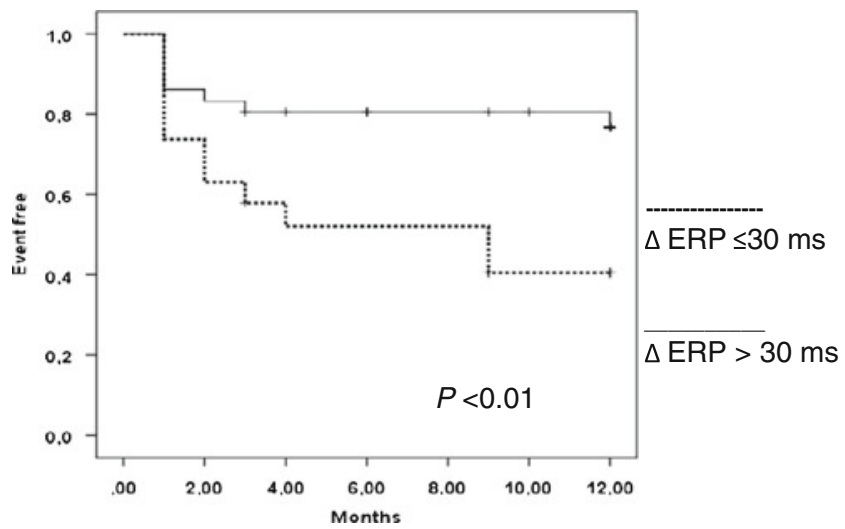

Fig. 4 Patients with post-procedural residual slow pathway. AVNRT recurrence-free event depending on $\Delta \mathrm{AV}$ nodal ERP. AVNRT atrioventricular nodal reentry tachycardia, $\triangle A V$ nodal $E R P$ difference between baseline and post-procedural nodal effective refractory period group and 19/71 (27\%) patients in the cryoablation group. Of these, one (5\%) and seven (36.8\%) patients, respectively, had documented arrhythmia recurrence $(p<0.05)$. Of note, in this study, isoproterenol was not uniformly used after ablation. In another study of subjects treated using only cryoenergy, Sandilands et al. [5] found that complete slow pathway conduction was associated with better long-term results. The procedure was considered successful if slow pathway suppression was achieved and/or in the presence of a single echo with AVNRT non-inducibility on isoproterenol. Recurrence rates were greater when slow pathway suppression was not achieved ( $8 / 12$ patients, $66.7 \%$ ), compared with complete slow pathway conduction (11/129 patients, $8.5 \%, P<0.0001)$. Recurrence was significantly more likely if echo beats were still present after cryoablation: 12/130 $(9.2 \%)$ patients with no recurrence vs. 7/19 (36.8\%) patients with recurrence $(P<0.0001)$. Other authors found no significant differences in terms of recurrence in the case of the persistence of dual AV nodal physiology [4].

In the present extended study conducted in a selected population of patients with baseline elicitable jump and inducible AVNRT, the persistence of a residual slow pathway was associated with a higher incidence of recurrence, but the presence of an associated single echo did not modify results. These results confirm those from our recent report [6]. Also, a longer total cryoapplication time and a residual jump both independently characterized patients with recurrence.

\subsection{Slow pathway modifications in patients treated} by cryoablation

There are scant data concerning slow pathway modification effects and the outcome in patients treated with cryoenergy [28]. In the present study, in the subgroup of patients without residual slow pathway conduction, the $\Delta$ AV ERP was not predictive of long-term results, whatever the cutoff used. The only factor correlated with recurrence was a prolonged cryoapplication time, which in this case may be considered as a marker of a difficult procedure, possibly linked to anatomical variants. Contrarily, in patients with slow pathway conduction at the end of the procedure, the ERP modification was correlated with recurrence. In the present study, a small variation of $\Delta \mathrm{AV} \mathrm{ERP} \leq 30 \mathrm{~ms}$ appeared predictive of a worse outcome. Cryoapplication time was 
globally prolonged in this subgroup, but it was not correlated with recurrence.

Recently, Posan et al. [29] showed that in patients with persistent slow pathway conduction after RF ablation, but no recurrent AVNRT, slow pathway ERP increased, while the difference between the fast and slow pathway ERP was reduced, thus decreasing the induction window of reentry [30]. The same operating mechanism could be extrapolated to cryoablation. In the present study, we did not systematically evaluate fast pathway ERP, and in order to avoid confounding factors, we excluded from analysis patients with unwanted transient first-degree degree AV block after cryoablation suggesting fast pathway damage.

AV nodal ERP can of course fluctuate during the procedure, depending on patient stress, vagal reactions, and the residual effect of given drugs. Therefore, we evaluated the AV nodal ERP at baseline and before isoproterenol infusion at the end of the procedure. A cutoff of $30 \mathrm{~ms}$ appeared the best predictor at Cox's model analysis.

\subsection{Clinical implications}

Based on the data presented here, slow pathway suppression must be the endpoint of cryoablation of AVNRT. Slow pathway persistence ("residual jump") can be tolerated if nodal ERP postcryoablation is prolonged more than $30 \mathrm{~ms}$, without AVNRT inducibility with and without isoproterenol.

\subsection{Study limitations}

In the present study, the recurrence rate was relatively high, probably linked to a long follow-up duration, a learning curve effect, the high number of operators with different levels of skill and training, and the fact that the main institution involved is a tertiary center where more difficult cases are generally addressed. Of note, the redo procedure rate was $7.9 \%$, strongly indicating that patients with recurrence who did not need further cryoablation had a substantial real-life improvement. Additionally, cryoablation of the slow pathway is a relatively new approach of catheter ablation for AVRNT compared to RF, and strategies to improve long-term results have been evaluated only in the recent past.

A parameter not evaluated in the present study was the "time to effect" during cryoablation, a phenomenon well known in RF ablation of accessory pathways, and also described in accessory pathway cryoablation in children [31].

\section{Conclusions}

Suppression of slow pathway conduction is the optimal endpoint for AVNRT cryoablation. In patients with a residual jump, an associated single echo did not modify results, while a small prolongation in post-cryo ERP $<30 \mathrm{~ms}$ was associated with a higher recurrence rate. Therefore, slow pathway persistence can be tolerated if nodal ERP is prolonged at the end of the procedure.

Conflict of interest All authors declared no conflict of interest and no financial interest.

Open Access This article is distributed under the terms of the Creative Commons Attribution License which permits any use, distribution, and reproduction in any medium, provided the original author(s) and the source are credited.

\section{References}

1. De Sisti, A., \& Tonet, J. (2011). Cryoablation of atrioventricular nodal reentrant tachycardia: a clinical review. Pacing and Clinical Electrophysiology, 10, 1-8.

2. Stern, J. D., Rolnitzky, L., Goldberg, J. D., Chinitz, L. A., Holmes, D. S., Bernstein, N. E., et al. (2011). Meta-analysis to assess the appropriate endpoint for slow pathway ablation of atrioventricular nodal reentrant tachycardia. Pacing and Clinical Electrophysiology, 34, 269-277.

3. Gupta, D., Al-Lamee, R. K., Earley, M. J., Kistler, P., Harris, S. J., Nathan, A. W., et al. (2006). Cryoablation compared with radiofrequency ablation for atrioventricular nodal re-entrant tachycardia: analysis of factors contributing to acute and follow-up outcome. Europace, 8, 1022-1026.

4. Chanani, N. K., Chiesa, N. A., Dubin, A. M., Avasarala, K., Van Hare, G. F., \& Collins, K. K. (2008). Cryoablation for atrioventricular nodal reentrant tachycardia in young patients: predictors of recurrence. Pacing and Clinical Electrophysiology, 31, 11521159.

5. Sandilands, A., Boreham, P., Pitts-Crick, J., \& Cripps, T. (2008). Impact of cryoablation catheter size on success rates in the treatment of atrioventricular nodal re-entry tachycardia in 160 patients with long-term follow-up. Europace, 10, 683-686.

6. De Sisti, A., Tonet, J., Amara, W., Raguin, D., Aouate, P., Gueffaf, F., et al. (2012). Correlations between long-term results after cryoablation for atrioventricular nodal reentry tachycardia and a residual jump associated or not with a single echo. Europace, 14, 261-266.

7. Jackman, W. M., Nakagawa, H., Heidbüchel, H., Beckman, K., McClelland, J., \& Lazzara, R. (1995). Three forms of atrioventricular nodal (junctional) re-entrant tachycardia: differential diagnosis, electrophysiological characteristics, and implications for anatomy of the re-entrant circuit. In D. P. Zipes \& J. Jalife (Eds.), Cardiac electrophysiology: from cell to bedside (pp. 620637). Philadelphia: Saunders.

8. Haïssaguerre, M., Gaita, F., Fischer, B., Commenges, D., Montserrat, P., d'Ivernois, C., et al. (1992). Elimination of atrioventricular nodal reentrant tachycardia using discrete slow potentials to guide application of radiofrequency energy. Circulation, 85, 2162-2175.

9. Kimman, G. P., Theuns, D. A. M. J., Szili-Torok, T., Scholten, M. F., Res, J. C., \& Jordaens, L. J. (2004). CRAVT: a prospective, randomized study comparing transvenous cryothermal and radiofrequency ablation in atrioventricular re-entrant tachycardia. European Heart Journal, 25, 2232-2237.

10. Zrenner, B., Dong, J., Schreieck, J., Deisenhofer, I., Estner, H., Luani, B., et al. (2004). Transvenous cryoablation versus 
radiofrequency ablation of the slow pathway for the treatment of atrioventricular nodal re-entrant tachycardia: a prospective randomized pilot study. European Heart Journal, 25, 2226-2231.

11. Jensen-Urstad, M., Tabrizi, F., Kenneback, G., Wredlert, C., Klang, C., \& Insulander, P. (2006). High success rate with cryomapping and cryoablation of atrioventricular nodal reentry tachycardia. Pacing and Clinical Electrophysiology, 29, 487489.

12. Collins, K. K., Dubin, A. M., Chiesa, N. A., Avasarala, K., \& Van Hare, G. F. (2006). Cryoablation versus radiofrequency ablation for treatment of pediatric atrioventricular reentrant tachycardia: initial experience with 4-mm cryocatheter. Heart Rhythm, 3, 564-570.

13. Khairy, P., Novak, P. G., Guerra, P. G., Greiss, I., Macle, L., Roy, D., et al. (2007). Cryothermal slow pathway modification for atrioventricular nodal reentrant tachycardia. Europace, 9, 909-914.

14. De Sisti, A., Tonet, J., Gueffaf, F., Touil, F., Leclercq, J. F., Aouate, P., et al. (2008). Effects of inadvertent atrioventricular block on clinical outcomes during cryoablation of the slow pathway in the treatment of atrioventricular nodal re-entrant tachycardia. Europace, 10, 1421-1427.

15. Avari, J. N., Jay, K. S., \& Rhee, E. K. (2008). Experience and results during transition from radiofrequency ablation to cryoablation for treatment of pediatric atrioventricular nodal reentrant tachycardia. Pacing and Clinical Electrophysiology, 31, 454-460.

16. Rivard, L., Dubuc, M., Guerra, P. G., Novak, P., Roy, D., Macle, L., et al. (2008). Cryoablation outcomes for AV nodal reentrant tachycardia comparing 4-mm versus 6-mm electrode-tip catheters. Heart Rhythm, 5, 230-234.

17. Chan, N. Y., Mok, N. S., Lau, C. L., Lo, Y. K., Choy, C. C., Lau, S. T., et al. (2009). Treatment of atrioventricular nodal re-entrant tachycardia by cryoablation with a $6 \mathrm{~mm}$-tip catheter vs. radiofrequency ablation. Europace, 11, 1065-1070.

18. Bastani, H., Schwieler, J., Insulander, P., Tabrizi, F., Braunschweig, F., Kennebäck, G., et al. (2009). Acute and long-term outcome of cryoablation therapy of typical atrioventricular nodal reentrant tachycardia. Europace, 11, 1077-1082.

19. LaPage, M. J., Saul, J. P., \& Reed, J. H. (2010). Long-term outcomes for cryoablation of pediatric patients with atrioventricular nodal reentrant tachycardia. The American Journal of Cardiology, 105, 1118-1121.

20. Drago, F., Russo, M. S., Silvetti, M. S., De Santis, A., Iodice, F., \& Naso Onofrio, M. T. (2010). Cryoablation of typical atrioventricular nodal reentrant tachycardia in children: six years' experience and follow-up in a single center. Pacing and Clinical Electrophysiology, 33, 475-481.
21. Gist, K., Tigges, C., Smith, G., \& Clark, J. (2011). Learning curve for zero-fluoroscopy catheter ablation of AVNRT: early versus late experience. Pacing and Clinical Electrophysiology, 34, 264-268.

22. Opel, A., Murray, S., Kamath, N., Dhinoja, M., Abrams, D., Sporton, S., et al. (2010). Cryoablation versus radiofrequency ablation for treatment of atrioventricular nodal reentrant tachycardia: cryoablation with 6-mm-tip catheters is still less effective than radiofrequency ablation. Heart Rhythm, 7, 340-343.

23. Deisenhofer, I., Zrenner, B., Yin, Y. H., Pitschner, H. F., Kuniss, M., Grossmann, G., et al. (2010). Cryoablation versus radiofrequency energy for the ablation of atrioventricular nodal reentrant tachycardia (the CYRANO Study): results from a large multicenter prospective randomized trial. Circulation, 122, 2239-2245.

24. Silver, E. S., Silva, J. N., Ceresnak, S. R., Chiesa, N. A., Rhee, E. K., Dubin, A. M., et al. (2010). Cryoablation with an 8-mm tip catheter for pediatric atrioventricular nodal reentrant tachycardia is safe and efficacious with a low incidence of recurrence. Pacing and Clinical Electrophysiology, 33, 681-686.

25. Schwagten, B., Knops, P., Janse, P., Kimman, G., Van Belle, Y., SziliTorok, T., et al. (2011). Long-term follow-up after catheter ablation for atrioventricular nodal reentrant tachycardia: a comparison of cryothermal and radiofrequency energy in a large series of patients. Journal of Interventional Cardiac Electrophysiology, 30, 55-61.

26. Calkins, H., Yong, P., Miller, J. M., Olshansky, B., Carlson, M., Saul, J. P., et al. (1999). Catheter ablation of accessory pathways, atrioventricular nodal reentrant tachycardia, and the atrioventricular junction: final results of a prospective, multicenter clinical trial. The Atakr Multicenter Investigators Group. Circulation, 99, 262-270.

27. Scheinman, M. M., \& Huang, S. (2000). The 1998 NASPE prospective catheter ablation registry. Pacing and Clinical Electrophysiology, 23, 1020-1028.

28. Márquez, M. F., Colín, L., Iturralde, P., Nava, S., González, E., Rodríguez, G., et al. (2005). La ablación criotérmica percutánea directa elimina la conducción de la vía lenta sin inducir ritmos ectópicos. Archivos de Cardiología de México, 75, 112-117.

29. Posan, E., Gula, L. J., Skanes, A. C., Krahn, A. D., Yee, R., Petrellis, B., et al. (2006). Characteristics of slow pathway conduction after successful AVNRT ablation. Journal of Cardiovascular Electrophysiology, 17, 847-851.

30. Wimmer, A. P., \& Shapiro, M. L. (2002). The "window" of slow pathway conduction after ablation and recurrence of atrioventricular nodal reentrant tachycardia. Journal of Interventional Cardiac Electrophysiology, 6, 51-57.

31. Drago, F., Russo, M. S., Silvetti, M. S., De Santis, A., \& Onofrio, M. T. (2009). 'Time to effect' during cryomapping: a parameter related to the long-term success of accessory pathways cryoablation in children. Europace, 11, 630-634. 